\title{
Nonperturbative flat direction decay
}

\author{
Anders Basbøll, ${ }^{1}$ David Maybury, ${ }^{2}$ Francesco Riva, ${ }^{2}$ and Stephen M. West ${ }^{2}$ \\ ${ }^{1}$ Department of Physics and Astronomy, University of Aarhus, Ny Munkegade, DK-8000 Aarhus C, Denmark \\ ${ }^{2}$ Rudolf Peierls Centre for Theoretical Physics, University of Oxford, 1 Keble Rd., Oxford OXI 3NP, United Kingdom
}

(Received 21 May 2007; published 12 September 2007)

\begin{abstract}
We argue that supersymmetric flat direction vacuum expectation values can decay nonperturbatively via preheating. Considering a toy $U(1)$ gauge theory, we explicitly calculate the scalar potential, in the unitary gauge, for excitations around several flat directions. We show that the mass matrix for the excitations has nondiagonal entries which vary with the phase of the flat direction vacuum expectation value. Furthermore, this mass matrix has zero eigenvalues whose eigenstates change with time. We show that these light degrees of freedom are produced copiously in the nonperturbative decay of the flat direction vacuum expectation value.
\end{abstract}

DOI: 10.1103/PhysRevD.76.065005

PACS numbers: 12.60.Jv, 98.80.Cq

\section{INTRODUCTION}

The scalar potential of the minimal supersymmetric standard model (MSSM) possesses a large number of $F$ and $D$-flat directions along which the scalar potential nearly vanishes $[1,2]$. These flat directions can have important cosmological consequences, including the generation of the baryon asymmetry of the Universe through the out-of-equilibrium $C P$ violating decay of coherent field oscillations along the flat directions themselves [3-7].

Recently, much interest has focused on the cosmological fate of flat direction vacuum expectation values (vev)s. In particular, it has been argued [8] that in realistic supersymmetric models, large flat direction vevs can persist long enough to delay thermalization after inflation and therefore lead to low reheat temperatures. Furthermore, it has also been asserted [9] that large flat direction vevs can prevent nonperturbative parametric resonant decay (preheating) of the inflaton since the inflaton decay products become sufficiently massive preventing preheating from ever becoming efficient. These arguments hold so long as the flat direction vevs do not rapidly decay - they must persist long enough so that they can delay thermalization and block inflaton preheating. In [10] it was claimed that nonperturbative decay can lead to a rapid depletion of the flat direction condensate and thus precludes the delay of thermalization after inflation. It was also concluded that in order for the flat direction to decay nonperturbatively the system requires more than one flat direction [10,11]. Finally, in [11] it was pointed out that even in the presence of multiple flat directions, some degree of fine-tuning was necessary to achieve flat direction decay.

An important aspect of this discussion centers on the issue of Nambu-Goldstone (NG) bosons. In general, supersymmetric flat directions are charged under the gauge group of the MSSM. Consequently, the flat direction vev will break some or all of the gauge symmetries of the theory and thus we expect the presence of the associated NG bosons. In calculating nonperturbative flat direction decays, [10] considers a gauged $U(1)$ model and constructs the mixing matrix for the excitations around the flat direction vev. The results in [10] show that in the single flat direction case, nonperturbative decay proceeds solely via a massless NG mode as only the NG mode mixes with the Higgs and all other massless moduli remain decoupled. Since the NG boson represents an unphysical gauge degree of freedom, it was concluded $[10,11]$ that no preheating occurs in the single flat direction case. As the appearance of a massless NG boson in the spectrum is a gauge dependent artifact, it remains unclear if the conclusions drawn about the system hold in the unitary gauge. In order to determine if flat direction vevs decay nonperturbatively into scalar degrees of freedom, the effect of the NG boson mixing in the scalar potential must first be removed. The process of removing the NG modes by switching to the unitary gauge changes the form of the mixing matrix among the leftover scalar degrees of freedom.

In this paper we consider toy models to demonstrate that, in the unitary gauge, the mixing matrix of the excitations around a flat direction vev permits preheating. Moreover, we find that flat direction decay depends on the number of dynamical, physical phases appearing in the flat direction vev. Specifically, a physical phase difference between two of the individual field vevs making up the flat direction is needed.

The outline of the rest of this paper proceeds as follows: first we explicitly construct - in the unitary gauge - the mass squared matrix arising from the $D$-terms of a toy gauged $U(1)$ model with three charged chiral superfields. We then present the formalism of preheating with multicomponent fields and show that preheating occurs for the light moduli associated with the flat direction. We then analyze the specific dynamics of the background field equations for the toy models examined. Finally we evolve the background field equations for one of the toy models to obtain quantitative results. 


\section{TOY MODEL WITH A GAUGED $U(1)$ SYMMETRY}

As an example, we examine a toy model which demonstrates the important features of supersymmetric flat direction vev decay. We introduce three complex superfields, ${ }^{1}$ $\Phi_{1}, \Phi_{2}$, and $\Phi_{3}$ charged under a $U(1)$ gauge group with charges $q_{1}=+1 / 2, q_{2}=-1$, and $q_{3}=+1 / 2$, respectively. The Lagrangian reads

$$
\mathcal{L}=\sum_{i=1}^{3} \frac{1}{2}\left|D_{\mu} \Phi_{i}\right|^{2}-V-\frac{1}{4} F_{\mu \nu}^{2},
$$

where $D_{\mu}=\partial_{\mu}-i q_{i} A_{\mu}$ denotes the covariant derivative. The potential we consider arises from the supersymmetric $D$-terms and has the form

$$
V=\frac{g^{2}}{2}\left(q_{1}\left|\Phi_{1}\right|^{2}+q_{2}\left|\Phi_{2}\right|^{2}+q_{3}\left|\Phi_{3}\right|^{2}\right)^{2},
$$

where $g$ is the gauge coupling of the $U(1)$ gauge symmetry. In the above, we have neglected contributions from supersymmetry (SUSY) breaking and from any nonrenormalizable terms arising from the superpotential. These contributions are highly model dependent and cloud the analysis we wish to present. A fully realistic model must include these additional contributions which can significantly affect the resulting particle production. The effects we investigate here do not depend on their inclusion in the quadratic part of the potential and so for clarity we neglect them. ${ }^{2}$

The potential in Eq. (2) admits several flat directions. Choosing one particular direction and including excitations around the vev we can write

$$
\begin{aligned}
& \Phi_{1}=\left(\varphi+\xi_{1}\right) e^{i\left(\sigma_{1}+\left(\theta_{1} / \varphi\right)\right),} \\
& \Phi_{2}=\left(\varphi+\xi_{2}\right) e^{i\left(\sigma_{2}+\left(\theta_{2} / \varphi\right)\right)}, \\
& \Phi_{3}=\left(\varphi+\xi_{3}\right) e^{i\left(\sigma_{3}+\left(\theta_{3} / \varphi\right)\right)},
\end{aligned}
$$

where $\sigma_{1,2,3}$ represent time-dependent phases of the vevs, $\varphi$ denotes the vev's time-dependent amplitude ${ }^{3}$ while $\xi_{1,2,3}$ and $\theta_{1,2,3}$ parametrize the six real scalar degrees of freedom corresponding to the excitations around the vevs. Note that the flat direction vev breaks the $U(1)$ gauge symmetry. Thus, out of the six real scalar degrees of freedom we expect one massive Higgs field and one massless NG boson, leaving four massless scalar degrees of freedom.

The kinetic terms for the scalar fields play an important role in this analysis. Their expansion in Eq. (1) includes the term

\footnotetext{
${ }^{1}$ We use $\Phi$ to denote both superfields and scalar components of superfields.

${ }^{2}$ SUSY breaking terms are needed for the computation of the evolution of the flat direction vev.

${ }^{3}$ Throughout this analysis we assume $\dot{\varphi} \ll \varphi \dot{\sigma}$.
}

$$
\mathcal{L} \supset-\varphi^{2} A_{0}\left(\dot{\sigma}_{1}-2 \dot{\sigma}_{2}+\dot{\sigma}_{3}\right)
$$

which has the form of a coupling between the gauge field and the background condensate. Terms of this type will feed into the equations of motion for the gauge field which, in turn, will have an effect on the equations of motion for the scalar excitations. By making a $U(1)$ gauge transformation on the vev of the form

$$
\left\langle\Phi_{i}\right\rangle \rightarrow\left\langle\Phi_{i}^{\prime}\right\rangle=e^{i q_{i} \lambda}\left\langle\Phi_{i}\right\rangle
$$

with

$$
\lambda=\frac{2 \sigma_{2}-\sigma_{1}-\sigma_{3}}{3},
$$

we can gauge this term away and avoid a complicated analysis of the kinetic terms. The resulting form of the vev reads

$$
\begin{gathered}
\left\langle\Phi_{1}\right\rangle=\varphi e^{i(\sigma+\gamma)}, \quad\left\langle\Phi_{2}\right\rangle=\varphi e^{i \sigma}, \\
\left\langle\Phi_{3}\right\rangle=\varphi e^{i(\sigma-\gamma)},
\end{gathered}
$$

where $\gamma=\left(\sigma_{1}-\sigma_{3}\right) / 2$ and $\sigma=\left(\sigma_{1}+\sigma_{2}+\sigma_{3}\right) / 3$ represent the two remaining independent physical phases. Following Kibble [12], we can write the fields in the unitary gauge as

$$
\begin{aligned}
& \Phi_{1}=\left(\varphi+\xi_{1}\right) e^{i\left(\sigma+\gamma+(\theta / \varphi \sqrt{2})+\left(\theta^{\prime} / \varphi \sqrt{3}\right)\right)}, \\
& \Phi_{2}=\left(\varphi+\xi_{2}\right) e^{i\left(\sigma+\left(\theta^{\prime} / \varphi \sqrt{3}\right)\right)}, \\
& \Phi_{3}=\left(\varphi+\xi_{3}\right) e^{i\left(\sigma-\gamma-(\theta / \varphi \sqrt{2})+\left(\theta^{\prime} / \varphi \sqrt{3}\right)\right.},
\end{aligned}
$$

where $\xi_{1,2,3}, \theta$ and $\theta^{\prime}$ denote the physical excitations - the $\mathrm{NG}$ boson has been removed. ${ }^{4}$ We choose the particular combination of field excitations appearing in Eq. (8) (the exponent, in particular) in order to retain canonically normalized kinetic terms.

On substituting the fields of Eq. (8) into the Lagrangian given in Eq. (1) and defining the vector $\Xi \equiv$ $\left(\xi_{1}, \xi_{2}, \xi_{3}, \theta, \theta^{\prime}\right)^{T}$, we find the quadratic terms

$$
\mathcal{L} \supset \frac{1}{2}\left|\partial_{\mu} \Xi\right|^{2}-\frac{1}{2} \Xi^{T} \mathcal{M}^{2} \Xi+\frac{1}{2} \dot{\Xi}^{T} U \Xi+\ldots,
$$

where the ellipses denote higher order terms and interactions. The matrix $U$ given in the last term in Eq. (9) reads

$$
U=\left(\begin{array}{ccccc}
0 & 0 & 0 & \frac{\dot{\sigma}+\dot{\gamma}}{\sqrt{2}} & \frac{\dot{\sigma}+\dot{\gamma}}{\sqrt{3}} \\
0 & 0 & 0 & 0 & \frac{\dot{\sigma}}{\sqrt{3}} \\
0 & 0 & 0 & \frac{-\dot{\sigma}+\dot{\gamma}}{\sqrt{2}} & \frac{\dot{\sigma}-\dot{\gamma}}{\sqrt{3}} \\
-\frac{\dot{\sigma}+\dot{\gamma}}{\sqrt{2}} & 0 & \frac{\dot{\sigma}-\dot{\gamma}}{\sqrt{2}} & 0 & 0 \\
-\frac{\dot{\sigma}+\dot{\gamma}}{\sqrt{3}} & -\frac{\dot{\sigma}}{\sqrt{3}} & \frac{-\boldsymbol{\sigma}+\dot{\gamma}}{\sqrt{2}} & 0 & 0
\end{array}\right),
$$

while the mass matrix for the physical excitations appears as

\footnotetext{
${ }^{4}$ This can be verified by expanding out the scalar kinetic terms which reveals the absence of terms of the form $A_{\mu} \partial^{\mu}(\ldots)$.
} 


$$
\mathcal{M}^{2}=(g \varphi)^{2}\left(\begin{array}{ccccc}
1 & -2 & 1 & 0 & 0 \\
-2 & 4 & -2 & 0 & 0 \\
1 & -2 & 1 & 0 & 0 \\
0 & 0 & 0 & 0 & 0 \\
0 & 0 & 0 & 0 & 0
\end{array}\right)=B \mathcal{M}_{d}^{2} B^{T}
$$

with eigenvalues $M_{1}^{2}=6(g \varphi)^{2}, M_{2}^{2}=M_{3}^{2}=M_{4}^{2}=M_{5}^{2}=$ 0 (the entries of the diagonal matrix $\mathcal{M}_{d}$ ). $B$ is an orthogonal matrix which diagonalizes $\mathcal{M}^{2}$ and $M_{1}$ corresponds to the mass of the physical Higgs field associated with the spontaneous breaking of the $U(1)$ symmetry. The four zero eigenvalues correspond to the massless excitations around the flat direction vev.

The last term in Eq. (9) appears as a consequence of the time dependence of the background - it represents a mixing between the fields $\xi_{1,2,3}, \theta, \theta^{\prime}$ and their time derivatives. The effect of these terms on the system becomes clear if we make field redefinitions that remove the mixed derivative terms. The resulting transformation leaves the system in an inertial frame in field space and leads to a time-dependent mass matrix. Defining $\Xi^{\prime}=A \Xi(A$ is orthogonal), we find the condition that $A$ must satisfy, in order for all the mixed derivative terms to cancel, to be

$$
\dot{A}^{T} A=U \text {. }
$$

The Lagrangian for the $\Xi^{\prime}$ system now reads

$$
\mathcal{L} \supset \frac{1}{2}\left|\partial_{\mu} \Xi^{\prime}\right|^{2}-\frac{1}{2} \Xi^{\prime T} \mathcal{M}^{\prime 2} \Xi^{\prime},
$$

where $\mathcal{M}^{\prime 2}=A \mathcal{M}^{2} A^{T}=A B \mathcal{M}_{d}^{2} B^{T} A^{T}=C \mathcal{M}_{d}^{2} C^{T}$ and $C=A B$. The matrix $C$ is an orthogonal time-dependent matrix, with columns corresponding to the eigenvectors of $\mathcal{M}^{\prime 2}$. We now have a system of scalar fields with canonically normalized kinetic terms and time-dependent eigenvectors.

The central point of this discussion centers precisely on the appearance of the time-dependent eigenvectors for the five scalar fields. This satisfies a necessary but not sufficient condition for preheating. In the next sections, we investigate the details of the nonperturbative production of the light scalar fields following the analysis of [13].

\section{NONPERTURBATIVE PRODUCTION OF PARTICLES}

Including gravity, the dynamics of the rescaled conformally coupled scalar fields, $\chi_{i}=a \Xi_{i}^{\prime}$, where $a$ denotes the scale factor and $\Xi_{i}^{\prime}$ the $i$ th component of the vector $\Xi^{\prime}$, are governed by the following equations of motion (sum over repeated indices is implied),

$$
\ddot{\chi}_{i}+\Omega_{i j}^{2}(t) \chi_{j}=0,
$$

where dots represent derivatives with respect to conformal time $t$, and

$$
\Omega_{i j}^{2}=a^{2} \mathcal{M}_{i j}^{\prime 2}+k^{2} \delta_{i j},
$$

where $k$ labels the comoving momentum. Using an orthogonal time-dependent matrix $C(t)$, we can diagonalize $\Omega_{i j}$ via $C^{T}(t) \Omega^{2}(t) C(t)=\omega^{2}(t)$, giving the diagonal entries $\omega_{j}^{2}(t)$. Terms of the form $\sim \varphi \dot{\sigma} \dot{\chi}$ arising from the kinetic terms do not affect the evolution of the nonzero $k$ quantum modes [14].

Once we have identified the basis in which the Hamiltonian appears diagonal (via the orthogonal matrix $C(t)$ ), the study of particle creation by the time-varying background proceeds as in $[13,15,16]$, which extends the results of [17]. Following [13], we assume that $\Omega_{i j}$ initially evolves adiabatically by assuming that the initial angular motion of the flat direction varies slowly. This assumption allows us to define adiabatically evolving mode functions with positive and negative frequency. We rewrite the quantum fields as mode expansions in terms of the mode functions and their associated creation/annihilation operators which allows us to define the initial vacuum. During the evolution, the entries of $\Omega_{i j}$ do not necessarily change adiabatically and consequently we must find new mode functions that satisfy Eq. (14). A new set of creation/ annihilation operators required to define the new vacuum can be related to the initial set using a Bogolyubov transformation with Bogolyubov coefficients $\alpha$ and $\beta$ (which denote matrices in the multifield case).

Initially $\alpha=\rrbracket$ and $\beta=0$ while the coupled differential equations (matrix multiplication implied)

$$
\begin{aligned}
& \dot{\alpha}=-i \omega \alpha+\frac{\dot{\omega}}{2 \omega} \beta-I \alpha-J \beta, \\
& \dot{\beta}=\frac{\dot{\omega}}{2 \omega} \alpha+i \omega \beta-J \alpha-I \beta
\end{aligned}
$$

govern the system's time evolution with the matrices $I$ and $J$ given by

$$
\begin{aligned}
& I=\frac{1}{2}\left(\sqrt{\omega} C^{T} \dot{C} \frac{1}{\sqrt{\omega}}+\frac{1}{\sqrt{\omega}} C^{T} \dot{C} \sqrt{\omega}\right), \\
& J=\frac{1}{2}\left(\sqrt{\omega} C^{T} \dot{C} \frac{1}{\sqrt{\omega}}-\frac{1}{\sqrt{\omega}} C^{T} \dot{C} \sqrt{\omega}\right) .
\end{aligned}
$$

Similarly to the single-field case it can be shown [13] that at any generic time the occupation number of the $i$ th bosonic eigenstate reads

$$
n_{i}(t)=\left(\beta^{*} \beta^{T}\right)_{i i} .
$$

As pointed out in $[10,13]$, there exists two sources of nonadiabaticity in the multifield scenario. The first source arises from the individual frequency time dependence and appears as the only source of nonadiabaticity in the singlefield case. The second source appears from the time dependence of the frequency matrix $\Omega_{i j}$ giving rise to terms in Eq. (16) proportional to $I$ and $J$. This second source 
provides the most important contribution in our analysis and gives rise to nonperturbative particle production.

Since initially $\alpha=\rrbracket$ and $\beta=0$, Eq. (16) shows that a nonvanishing matrix $J$ is a necessary condition to obtain $\dot{\beta} \neq 0$ and hence $n_{i}(t) \neq 0$. In general, we have

$$
C^{T} \dot{C}=B^{T} A^{T} \dot{A} B=-B^{T} U B
$$

where $A, B$, and $U$ were defined in the previous section. For the toy $U(1)$ example outlined above, $J$ is a $5 \times 5$ matrix in the $\chi_{i}$ basis with nonvanishing components

$$
J_{1,2}=J_{2,1}=\frac{k-\sqrt{k^{2}+M_{1}^{2}}}{2 \sqrt{3 k}\left(k^{2}+M_{1}^{2}\right)^{1 / 4}} \dot{\gamma}
$$

where $M_{1}$ denotes the mass of the heavy Higgs field. These entries in the matrix $J$ link the eigenstate of the Higgs ( $i=$ $1)$ with one of the light eigenstates $(i=2)$. We see that in the toy $U(1)$ model, preheating can occur provided that $\dot{\gamma} \neq 0$. We address this point in a later section.

\section{MULTIPLE VEV AMPLITUDES}

We can extend the analysis of the previous sections by allowing the magnitudes of the individual field vevs to differ from one another. As above, we consider the case with three complex superfields charged under a $U(1)$ gauge group with charges $q_{1}=+1 / 2, q_{2}=-1$, and $q_{3}=$ $+1 / 2$, respectively, and with the scalar potential given in Eq. (2). We can write the flat direction with the following vev

$$
\begin{gathered}
\left\langle\Phi_{1}\right\rangle=\varphi_{1} e^{i \sigma_{1}}, \quad\left\langle\Phi_{2}\right\rangle=\frac{1}{\sqrt{2}}\left(\varphi_{1}^{2}+\varphi_{2}^{2}\right)^{1 / 2} e^{i \sigma_{2}} \\
\left\langle\Phi_{3}\right\rangle=\varphi_{2} e^{i \sigma_{3}}
\end{gathered}
$$

By substituting the above into the potential given in Eq. (2), it can readily be shown that the configuration satisfies $D$-flatness. Expanding around this vev we have

$\Phi_{1}=\left(\varphi_{1}+\xi_{1}\right) e^{i\left(\sigma_{1}+\left(\theta_{1} / \varphi_{1}\right)\right)}$,

$\Phi_{2}=\left(\frac{1}{\sqrt{2}}\left(\varphi_{1}^{2}+\varphi_{2}^{2}\right)^{1 / 2}+\xi_{2}\right) e^{i\left(\sigma_{2}+\left(\sqrt{2} \theta_{2} /\left(\varphi_{1}^{2}+\varphi_{2}^{2}\right)^{1 / 2}\right)\right)}$,

$\Phi_{3}=\left(\varphi_{2}+\xi_{3}\right) e^{i\left(\sigma_{3}+\left(\theta_{3} / \varphi_{2}\right)\right)}$,

where the fields $\xi_{1,2,3}$ and $\theta_{1,2,3}$ represent the excitations around the vevs. As in the previous case, we can use a gauge transformation to remove a phase from the vev structure that ensures the absence of terms of the form appearing in Eq. (4). The form of the vev in this case becomes,

$$
\begin{gathered}
\left\langle\Phi_{1}\right\rangle=\varphi_{1} e^{i\left(\sigma+\left(\varphi_{2} / \varphi_{1}\right) \gamma\right)}, \quad\left\langle\Phi_{2}\right\rangle=\frac{1}{\sqrt{2}}\left(\varphi_{1}^{2}+\varphi_{2}^{2}\right)^{1 / 2} e^{i \sigma}, \\
\left\langle\Phi_{3}\right\rangle=\varphi_{2} e^{i\left(\sigma-\left(\varphi_{1} / \varphi_{2}\right) \gamma\right)},
\end{gathered}
$$

where $\sigma$ and $\gamma$ represent two independent phases. ${ }^{5}$

In the unitary gauge, a form that preserves the canonically normalized kinetic terms reads,

$$
\begin{aligned}
\Phi_{1}= & \left(\varphi_{1}+\xi_{1}\right) \\
& \times e^{i\left(\sigma+\left(\varphi_{2} / \varphi_{1}\right) \gamma+\left[\left(\theta \varphi_{2}+\theta^{\prime} \varphi_{1}(2 / 3)^{1 / 2}\right) /\left(\varphi_{1}\left(\varphi_{1}^{2}+\varphi_{2}^{2}\right)^{1 / 2}\right)\right]\right)}, \\
\Phi_{2}= & \left(\frac{1}{\sqrt{2}}\left(\varphi_{1}^{2}+\varphi_{2}^{2}\right)^{1 / 2}+\xi_{2}\right) e^{i\left(\sigma+\left(\theta^{\prime}(2 / 3)^{1 / 2} /\left(\varphi_{1}^{2}+\varphi_{2}^{2}\right)^{1 / 2}\right)\right)}, \\
\Phi_{3}= & \left(\varphi_{2}+\xi_{3}\right) \\
& \times e^{i\left(\sigma-\left(\varphi_{1} / \varphi_{2}\right) \gamma-\left[\left(\theta \varphi_{1}-\theta^{\prime} \varphi_{2}(2 / 3)^{1 / 2}\right) /\left(\varphi_{2}\left(\varphi_{1}^{2}+\varphi_{2}^{2}\right)^{1 / 2}\right)\right]\right)},
\end{aligned}
$$

where $\xi_{1,2,3}, \theta$ and $\theta^{\prime}$ label the physical excitations around the vev once the NG boson has been gauged away. The resulting spectrum consists of one Higgs field with mass $M_{1}^{2}=3 g^{2}\left(\varphi_{1}^{2}+\varphi_{2}^{2}\right)$, and four massless scalar fields.

We proceed, as before, by diagonalizing the kinetic terms and evaluating the $J$ matrix given in Eq. (18). The nonvanishing entries of the $J$ matrix are

$$
J_{1,2}=J_{2,1}=\frac{k-\sqrt{k^{2}+M_{1}^{2}}}{2 \sqrt{3 k}\left(k^{2}+M_{1}^{2}\right)^{1 / 4}} \dot{\gamma},
$$

which demonstrates that in this case preheating can take place provided that $\dot{\gamma} \neq 0$.

It is instructive to compare the two cases considered thus far. The first flat direction contained a single vev amplitude, the second contained two independent vev amplitudes. The final result, however, is the same for both cases. This demonstrates a simple property of flat direction vev decay: the determining factor is not the number of flat directions present in the system, but the number of fields that have vevs. In particular, a necessary (but not sufficient) condition for nonperturbative production of particles is the existence of at least one relative physical and dynamical phase between the field vevs that constitute the flat direction.

\section{DYNAMICS OF THE VEV PHASES}

We now demonstrate that both physical phases are in general dynamical. In our particular toy example the cancellation of $U(1)^{3}$ and mixed $U(1)$-gravitational anomalies requires that we extend the field content of our model by including three additional complex superfields, $\Phi_{4}, \Phi_{5}$,

\footnotetext{
${ }^{5}$ Again we have applied the limit $\dot{\varphi} \ll \varphi \dot{\sigma}, \varphi \dot{\gamma}$. If we do not apply this limit the gauge transformation parameter $(\lambda)$ needed to remove the linear term in $A_{0}$ can be found by integrating the coefficient of the $A_{0}$ term with respect to time. This is in general complicated and we choose to assume that $\varphi$ is varying very slowly with time.
} 
and $\Phi_{6}$. We assign the $U(1)$ charges and $R$-Parity $\left(R_{p}\right)$ as follows:

\begin{tabular}{lllllll}
\hline \hline & $\Phi_{1}$ & $\Phi_{2}$ & $\Phi_{3}$ & $\Phi_{4}$ & $\Phi_{5}$ & $\Phi_{6}$ \\
\hline$U(1)$ & $1 / 2$ & -1 & $1 / 2$ & $-1 / 2$ & 1 & $-1 / 2$ \\
$R_{p}$ & + & - & + & - & + & - \\
\hline \hline
\end{tabular}

This choice of $R_{p}$ assignments forbids the superpotential term $\Phi_{1} \Phi_{2} \Phi_{3}$, thus preserving $F$-flatness. There exist several possible flat directions for this particular field content. We assume that vevs for only $\Phi_{1}, \Phi_{2}$, and $\Phi_{3}$ are turned on, leaving $\Phi_{4}, \Phi_{5}$, and $\Phi_{6}$ with no vevs. With this assumption, the lowest dimension gauge invariant operators which have vevs are

$$
\mathcal{O}_{1}=\Phi_{1} \Phi_{2} \Phi_{3}, \quad \mathcal{O}_{2}=\Phi_{1}^{2} \Phi_{2}, \quad \text { and } \quad \mathcal{O}_{3}=\Phi_{2} \Phi_{3}^{2} .
$$

Note that the last two operators depend on both physical phases, $\sigma$ and $\gamma$. Using these operators, the lowest dimension terms appearing in the scalar potential, which are $R_{p}$ invariant and phase dependent, arise as soft SUSY breaking $A$-terms and appear as

$$
V \supset \sum_{i, j} \frac{A_{i j} m_{s}}{M^{3}} \mathcal{O}_{i} \mathcal{O}_{j}+\text { H.c., }
$$

where $M$ denotes the cutoff scale of the theory (e.g. the Planck mass or grand unified theory (GUT) scale), $m_{s}$ represents the scale of the SUSY breaking, and $A_{i j}$ label dimensionless coefficients of order one. Lower order phaseindependent interactions will also contribute to the lifting of the flat direction and have the generic forms

$$
V \supset \sum_{i} \frac{m_{i}^{2}}{2}\left|\Phi_{i}\right|^{2}+\sum_{i, j} \frac{\lambda_{i j}}{8}\left|\Phi_{i}\right|^{2}\left|\Phi_{j}\right|^{2},
$$

where $m_{i}^{2}$ denote the soft SUSY breaking masses, and the second terms arise from loop corrections with $\lambda_{i, j} \sim$ $g^{4} m_{s}^{2} / \varphi^{2}$ (see for example [3] for similar loop induced terms). The potential for the single flat direction amplitude case considered in Sec. II, using the vev form shown in Eq. (7), becomes

$$
\begin{aligned}
V \supset & \frac{m_{1}^{2}+m_{2}^{2}+m_{3}^{2}}{2} \varphi^{2}+\frac{\lambda^{\prime}}{4} \varphi^{4} \\
& +\varphi^{6}\left(A_{11}^{\prime} e^{i 6 \sigma}+A_{12}^{\prime} e^{i(6 \sigma+2 \gamma)}\right. \\
& \left.+A_{13}^{\prime} e^{i(6 \sigma-2 \gamma)}+A_{22}^{\prime} e^{i(6 \sigma+4 \gamma)}+A_{33}^{\prime} e^{i(6 \sigma-4 \gamma)}\right),
\end{aligned}
$$

where $A_{i j}^{\prime}$ and $\lambda^{\prime}$ denote combinations of the couplings discussed above. The potential for the multiple vev amplitude case will be very similar with the obvious changes of vev amplitudes. The phase-dependent terms in the potential provide nontrivial dynamics for the phases $\sigma$ and $\gamma$ and will in general lead to $\dot{\gamma} \neq 0$ and therefore a nonvanishing $J$ matrix. As discussed above, the appearance of a nonvanishing $J$ matrix can lead to the nonperturbative production of particles by the rotating flat direction: the condensate can decay via preheating.

\section{TWO INDEPENDENT FLAT DIRECTIONS}

A further instructive toy model consists of two independent flat directions existing simultaneously. We consider four chiral superfields $\Phi_{1}, \Phi_{2}, \Phi_{3}$, and $\Phi_{4}$ charged under a gauged $U(1)$ symmetry with charges $\pm q_{1}$ and $\pm q_{2}$, respectively. The potential arising from the $D$-terms reads

$$
V=\frac{g^{2}}{8}\left(q_{1}\left|\Phi_{1}\right|^{2}-q_{1}\left|\Phi_{2}\right|^{2}+q_{2}\left|\Phi_{3}\right|^{2}-q_{2}\left|\Phi_{4}\right|^{2}\right)^{2} .
$$

Although this toy model has been examined previously in [10], applying the methods outlined in the first sections of this paper helps establish the important properties of the model. The potential in Eq. (31) admits flat direction vevs of the following forms

$$
\begin{aligned}
\left\langle\Phi_{1}\right\rangle & =\varphi_{1} e^{i \tilde{\sigma}_{1}}, & \left\langle\Phi_{2}\right\rangle & =\varphi_{1} e^{i \tilde{\sigma}_{2}}, \\
\left\langle\Phi_{3}\right\rangle & =\varphi_{2} e^{i \tilde{\sigma}_{3}}, & \left\langle\Phi_{4}\right\rangle & =\varphi_{2} e^{i \tilde{\sigma}_{4}} .
\end{aligned}
$$

We can write the excitations around the vevs as

$$
\begin{aligned}
& \Phi_{1}=\left(\varphi_{1}+\xi_{1}\right) e^{i\left(\tilde{\sigma}_{1}+\left(\theta_{1} / \varphi_{1}\right)\right)}, \\
& \Phi_{2}=\left(\varphi_{1}+\xi_{2}\right) e^{i\left(\tilde{\sigma}_{2}+\left(\theta_{2} / \varphi_{1}\right)\right)}, \\
& \Phi_{3}=\left(\varphi_{2}+\xi_{3}\right) e^{i\left(\tilde{\sigma}_{3}+\left(\theta_{3} / \varphi_{2}\right)\right)}, \\
& \Phi_{4}=\left(\varphi_{2}+\xi_{4}\right) e^{i\left(\tilde{\sigma}_{4}+\left(\theta_{4} / \varphi_{2}\right)\right)} .
\end{aligned}
$$

As before we can make a gauge transformation and remove one phase in such a way that terms of the form shown in Eq. (4) vanish. The final form appears as

$$
\begin{aligned}
& \left\langle\Phi_{1}\right\rangle=\varphi_{1} e^{i\left(\sigma_{1}+\gamma\left(\varphi_{2} / q_{1} \varphi_{1}\right)\right)}, \\
& \left\langle\Phi_{2}\right\rangle=\varphi_{1} e^{i\left(\sigma_{1}-\gamma\left(\varphi_{2} / q_{1} \varphi_{1}\right)\right),} \\
& \left\langle\Phi_{3}\right\rangle=\varphi_{2} e^{i\left(\sigma_{2}+\gamma\left(\varphi_{1} / q_{2} \varphi_{2}\right)\right)}, \\
& \left\langle\Phi_{4}\right\rangle=\varphi_{2} e^{i\left(\sigma_{2}-\gamma\left(\varphi_{1} / q_{2} \varphi_{2}\right)\right)},
\end{aligned}
$$

demonstrating the existence of three physical phases. Transforming into the unitary gauge, we can write the excitations around the vevs as

$$
\begin{aligned}
& \Phi_{1}=\left(\varphi_{1}+\xi_{1}\right) e^{i\left(\sigma_{1}+\gamma\left(\varphi_{2} / q_{1} \varphi_{1}\right)+\theta\left(1 / \sqrt{2} \varphi_{1}\right)+\theta^{\prime \prime}\left(q_{2} \varphi_{2} / \varphi^{\prime} \varphi_{1}\right)\right)} \\
& \Phi_{2}=\left(\varphi_{1}+\xi_{2}\right) e^{i\left(\sigma_{1}-\gamma\left(\varphi_{2} / q_{1} \varphi_{1}\right)+\theta\left(1 / \sqrt{2} \varphi_{1}\right)-\theta^{\prime \prime}\left(q_{2} \varphi_{2} / \varphi^{\prime} \varphi_{1}\right)\right)} \\
& \Phi_{3}=\left(\varphi_{2}+\xi_{3}\right) e^{i\left(\sigma_{2}-\gamma\left(\varphi_{1} / q_{2} \varphi_{2}\right)+\theta^{\prime}\left(1 / \sqrt{2} \varphi_{2}\right)-\theta^{\prime \prime}\left(q_{1} \varphi_{1} / \varphi^{\prime} \varphi_{2}\right)\right)} \\
& \Phi_{4}=\left(\varphi_{2}+\xi_{4}\right) e^{i\left(\sigma_{2}+\gamma\left(\varphi_{1} / q_{2} \varphi_{2}\right)+\theta^{\prime}\left(1 / \sqrt{2} \varphi_{2}\right)+\theta^{\prime \prime}\left(q_{1} \varphi_{1} / \varphi^{\prime} \varphi_{2}\right)\right)}
\end{aligned}
$$

where $\varphi^{\prime}=\sqrt{2}\left(q_{1} \varphi_{1}^{2}+q_{2} \varphi_{2}^{2}\right)^{1 / 2}$. The spectrum in this case consists of one massive Higgs particle and six massless scalar fields (the NG has been gauged away). Again, we must diagonalize the kinetic terms. Applying the necessary field redefinitions we are able to evaluate the $J$ matrix. The nonvanishing $J$ matrix elements read 
$J_{1,2}=J_{2,1}=\frac{k-\sqrt{k^{2}+M_{1}^{2}}}{\sqrt{k}\left(k^{2}+M_{1}^{2}\right)^{1 / 4}} \frac{q_{1} q_{2}}{\varphi^{\prime 2}}\left(\dot{\sigma}_{1}-\dot{\sigma}_{2}\right) \varphi_{1} \varphi_{2}$,

$J_{1,3}=J_{3,1}=\frac{k-\sqrt{k^{2}+M_{1}^{2}}}{\sqrt{2 k}\left(k^{2}+M_{1}^{2}\right)^{1 / 4}} \frac{\dot{\gamma} \varphi_{2}}{\varphi^{\prime}}$,

$J_{1,4}=J_{4,1}=\frac{k-\sqrt{k^{2}+M_{1}^{2}}}{\sqrt{2 k}\left(k^{2}+M_{1}^{2}\right)^{1 / 4}} \frac{\dot{\gamma} \varphi_{1}}{\varphi^{\prime}}$,

which depend on the relative phases between the field vevs. We should point out that only the Higgs eigenstate $(i=1)$ is distinguishable. The other indices label the light fields which at this level are all massless. Preheating is again possible provided two of the phases have nonzero time derivatives. Using the particular case with $q_{1}=q_{2}$, we can write the scalar potential (see the appendix for details) yielding the terms

$$
\begin{aligned}
V= & \frac{1}{2}\left(m_{1}^{2}+m_{2}^{2}\right) \varphi_{1}^{2}+\frac{1}{2}\left(m_{3}^{2}+m_{4}^{2}\right) \varphi_{2}^{2} \\
& +\frac{A_{1}}{8} \frac{m_{s}}{M} \varphi_{1}^{4} e^{i 4 \sigma_{1}}+\frac{A_{2}}{8} \frac{m_{s}}{M} \varphi_{2}^{4} e^{i 4 \sigma_{2}} \\
& +\frac{A_{3}}{8} \frac{m_{s}}{M} \varphi_{1}^{2} \varphi_{2}^{2} e^{i 2\left(\sigma_{1}+\sigma_{2}+\gamma\left(\left(\varphi_{2}^{2}-\varphi_{1}^{2}\right) / \varphi_{2} \varphi_{1}\right)\right)} \\
& +\frac{A_{4}}{8} \frac{m_{s}}{M} \varphi_{1}^{2} \varphi_{2}^{2} e^{2 i\left(\sigma_{1}+\sigma_{2}-\gamma\left(\left(\varphi_{2}^{2}-\varphi_{1}^{2}\right) / \varphi_{2} \varphi_{1}\right)\right)}+\ldots
\end{aligned}
$$

Clearly, nontrivial dynamics exist for the phases $\gamma, \sigma_{1}$, and $\sigma_{2}$.

\section{NUMERICAL ANALYSIS}

As a proof-of-principle that achieves quantitative results, we numerically analyze the model described in Sec. VI. We use a simplified version of the potential appearing in Eq. (37), confining ourselves to the potential

$$
\begin{aligned}
V= & \frac{1}{2} m_{\varphi_{1}}^{2} \varphi_{1}^{2}+\frac{1}{2} m_{\varphi_{2}}^{2} \varphi_{2}^{2}+\frac{A_{1}}{8} \frac{m_{s}}{M} \varphi_{1}^{4} e^{i 4 \sigma_{1}} \\
& +\frac{A_{2}}{8} \frac{m_{s}}{M} \varphi_{2}^{4} e^{i 4 \sigma_{2}}+\text { H.c. },
\end{aligned}
$$

where $m_{\varphi_{1}}^{2}=m_{1}^{2}+m_{2}^{2}, m_{\varphi_{2}}^{2}=m_{3}^{2}+m_{4}^{2}$. This potential decouples the equations of motion for $\gamma, \sigma_{1}$, and $\sigma_{2}$. The equation of motion for $\gamma$ reduces to $\ddot{\gamma}=0$, and with the choice of initial conditions, $\dot{\gamma}=0$, the effects of $\gamma$ on preheating are removed. Our simplified potential allows us to numerically evolve the classical evolution of the flat direction vevs and analyze particle production in a selfconsistent background. We also make the simplifying assumption setting $A_{1}=A_{2}=\lambda \frac{M}{m_{s}}$ in Eq. (37). Again, we stress that we use this grossly simplified potential simply to demonstrate the quantitative behavior of the toy model class.

Measuring the conformal time in units of $\tau \rightarrow f t$ with $f=g \varphi_{1_{0}}$ and using the rescaled flat direction vev ampli- tudes

$$
\varphi_{1}=\frac{\varphi_{1_{0}}}{a} F_{1}, \quad \varphi_{2}=\frac{\varphi_{2_{0}}}{a} F_{2},
$$

we find the background equations,

$$
\begin{array}{r}
F_{1}^{\prime \prime}+\left[\frac{\mu_{1}^{2} a^{2}}{2}-\sigma_{1}^{\prime 2}-\frac{a^{\prime \prime}}{a}\right] F_{1}+\frac{\lambda F_{1}^{3}}{2 g^{2}} \cos \left(4 \sigma_{1}\right)=0, \\
F_{2}^{\prime \prime}+\left[\frac{\mu_{2}^{2} a^{2}}{2}-\sigma_{2}^{\prime 2}-\frac{a^{\prime \prime}}{a}\right] F_{2}+\frac{\lambda F_{2}^{3}}{2 g^{2}}\left(\frac{\varphi_{2_{0}}}{\varphi_{1_{0}}}\right)^{2} \cos \left(4 \sigma_{2}\right)=0,
\end{array}
$$

where a prime represents a derivative with respect to $\tau$ and

$$
\begin{array}{r}
\sigma_{1}^{\prime \prime}+2 \sigma_{1}^{\prime} \frac{F_{1}^{\prime}}{F_{1}}-\frac{\lambda}{2 g^{2}} F_{1}^{2} \sin \left(4 \sigma_{1}\right)=0, \\
\sigma_{2}^{\prime \prime}+2 \sigma_{2}^{\prime} \frac{F_{2}^{\prime}}{F_{2}}-\frac{\lambda}{2 g^{2}}\left(\frac{\varphi_{2_{0}}}{\varphi_{1_{0}}}\right)^{2} F_{2}^{2} \sin \left(4 \sigma_{2}\right)=0
\end{array}
$$

describes the motion of the flat direction vevs; $\mu_{1}=$ $m_{\varphi_{1}} / f, \mu_{2}=m_{\varphi_{2}} / f$. The scale factor evolves as,

$$
\begin{aligned}
\frac{a^{\prime \prime}}{a}= & -\frac{a^{\prime 2}}{a^{2}}+\frac{1}{2}\left[f _ { p } ^ { 2 } \left\{\mu_{1}^{2} F_{1}^{2}+\frac{\lambda}{2 g^{2}} \frac{F_{1}^{4}}{a^{2}} \cos \left(4 \sigma_{1}\right)+\mu_{2}^{2}\left(\frac{\varphi_{2_{0}}}{\varphi_{1_{0}}}\right)^{2} F_{2}^{2}\right.\right. \\
& \left.\left.+\frac{\lambda}{2 g^{2}}\left(\frac{\varphi_{2_{0}}}{\varphi_{1_{0}}}\right)^{4} \frac{F_{2}^{4}}{a^{2}} \cos \left(4 \sigma_{2}\right)\right\}+\frac{a^{2} \rho_{\psi}}{M_{\mathrm{pl}}^{2} f^{2}}\right],
\end{aligned}
$$

where $\rho_{\psi}$ is the energy density of the inflaton field and $f_{p}=\varphi_{1_{0}} / M_{\mathrm{pl}}$ is set to $f_{p}=0.1$ in our numerics. We also take $\mu_{1}=10^{-2}, \mu_{2}=10^{-2} / 2$, and $\lambda=\mu_{1}^{2}$ for computational ease. As initial conditions, we start the flat direction at rest, such that $\left(\varphi_{1,2} \exp \left(i \sigma_{1,2}\right)\right)^{\prime}=0$. We choose to set initially $F_{1,2}=1, \sigma_{1,2}=0.05, \sigma_{1,2}^{\prime}=0, a=1$, and $a^{\prime}=$ $\mu_{1}$, which implies $F_{1,2}^{\prime}=a^{\prime}=\mu_{1}$. While these initial conditions do not present a realistic case (where $\mu \sim$ $10^{-14}$ and $F_{1,2}^{\prime} \gg \mu_{1}$ ), they do provide a numerical proof-of-principle similar to [10].

Initially the flat direction vevs correspond to a condensate of coherent particles with vanishing momentum. The motion of these vevs, described by Eqs. (40) and (41), and the interactions described in the previous section, cause the rapid decay of this condensate into a decoherent state of particles. Figure 1 shows the occupation numbers, $n_{i}(t)$, of these light particles as a function of conformal time: the exponential growth of these functions signals the exponentially fast decay of the flat direction vev. The two line types, solid and dashed, represent the ratios $\varphi_{2_{0}} / \varphi_{1_{0}}=1,0.1$, respectively. We see that preheating occurs over a wide range of the ratio $\varphi_{2_{0}} / \varphi_{1_{0}}$. In this numerical example, preheating effects do not vanish until $\varphi_{2_{0}} / \varphi_{1_{0}} \lesssim 10^{-2}$. Figure 2 displays the resulting spectrum for one of the light fields, we see that production of higher momentum modes becomes kinematically suppressed.

We must stress that effects of SUSY breaking terms in the Lagrangian equation (1) will significantly affect the 


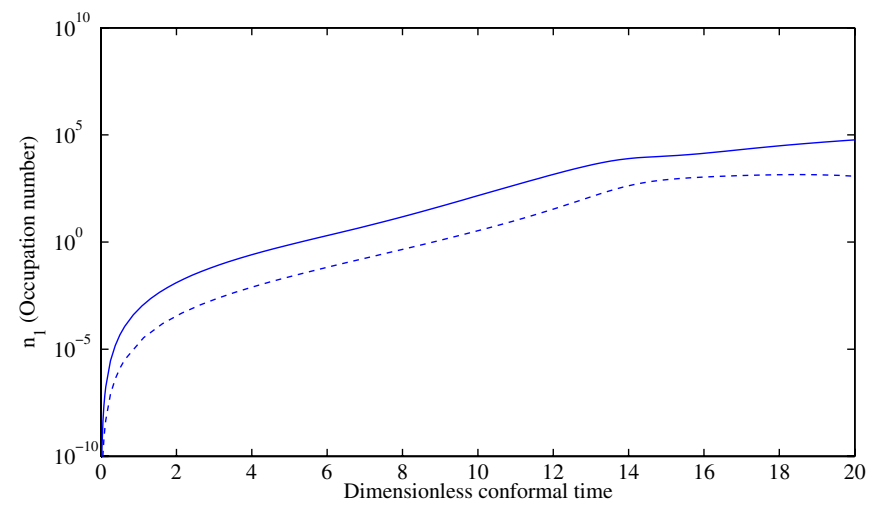

FIG. 1 (color online). Occupation number for one of the excited fields as a function of dimensionless conformal time, obtained using Eq. (19) after numerically integrating the background field equations and Bogolyubov matrices; $k=\mu_{1} / 3 \times$ $10^{5}$, other parameters as explained in the text. The solid lines represent preheated fields with $\varphi_{2_{0}} / \varphi_{1_{0}}=1$ while the dashed lines indicate the preheated fields with $\varphi_{2_{0}} / \varphi_{1_{0}}=0.1$.

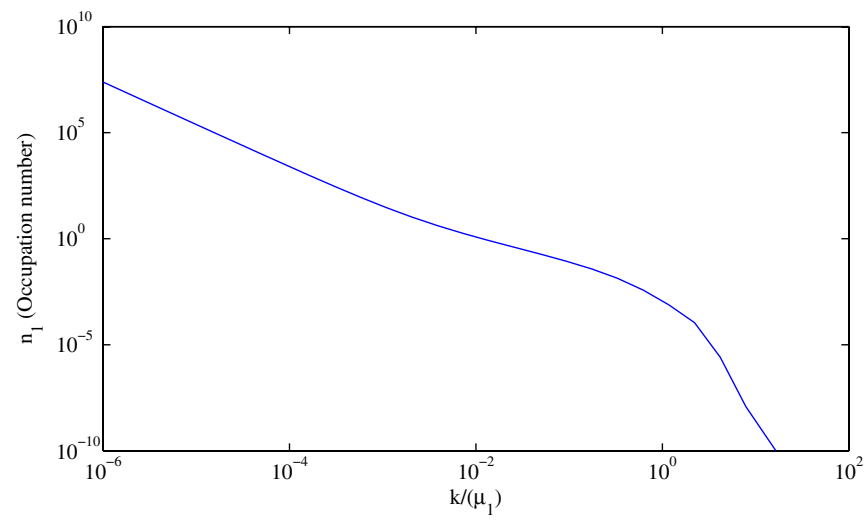

FIG. 2 (color online). Occupation number as a function of comoving momentum obtained as for Fig. 1, with $\varphi_{2_{0}} / \varphi_{1_{0}}=$ 0.5 at time $t=20$.

amount of particle production produced by the rotating condensate. A mass term for the light fields translates into a momentum shift in Eq. (15) and this corresponds to a kinematic suppression of the modes [10]. A realistic model involving MSSM flat directions will in general contain many SUSY breaking terms and nonrenormalizable operators, creating large model dependencies in the precise determination of the momentum shift. Consequently, we leave such a study to future work.

\section{CONCLUSIONS}

The cosmological fate of flat directions provides a major ingredient for the history of the early Universe. Flat directions can provide mechanisms for generating the baryon asymmetry of the Universe and can play an important role in reheating after inflation. Our analysis stresses the use of the unitary gauge in which the physical content of the theory becomes manifest. By transforming to the unitary gauge, complications arising from massless NG modes in the mixing of the excitations around the flat direction vev are removed. The mixing matrix in this gauge defines the mass eigenstates of the physical scalar fields and determines if nonperturbative decay is possible. Since the mass matrix in the unitary gauge can contain time-dependent mixing among all fields, one of the necessary conditions for preheating can be satisfied.

Two further crucial conditions for preheating in our analysis center on the existence of physical relative phases between the field vevs that make up the flat direction(s) and that these phases possess nontrivial dynamics during the early Universe. The first of these conditions generally becomes satisfied if the difference between the number fields that acquire a vev and the number of broken diagonal generators is larger than 1-every diagonal generator removes one unphysical phase. The second condition generally becomes satisfied if terms which explicitly depend on the phase differences appear in the scalar potential. The existence of gauge invariant products of background fields exhibiting this phase dependence represents the crucial ingredient and determines the phase dependence in the scalar potential. Once these conditions are satisfied, the flat direction condensate can decay nonperturbatively via preheating.

\section{ACKNOWLEDGMENTS}

We thank R. Allahverdi and J. March-Russell for many useful discussions. We are also grateful to I. Aitchison, S. Hannestad, P. Iafelice, G. Ross, S. Sarkar, D. Ghilencea, M. Schvellinger, and M. Sloth for helpful discussions. A. B. wishes to thank Oxford University for hospitality during the course of this work, D. M. is supported by the NSERC Canada, F. R. by Merton College. This work was partially supported by the EU 6th Framework Marie Curie Research and Training network "UniverseNet" (MRTN-CT-2006035863).

\section{APPENDIX}

In this appendix we specify a simple model to justify the form of the potential given in Eq. (37). We have the following field content with $U(1)$ charges and $R_{p}$ assignments,

\begin{tabular}{lllll}
\hline \hline & $\Phi_{1}$ & $\Phi_{2}$ & $\Phi_{3}$ & $\Phi_{4}$ \\
\hline$U(1)$ & 1 & -1 & 1 & -1 \\
$R_{p}$ & - & + & - & + \\
\hline \hline
\end{tabular}

The lowest dimension gauge invariant operators are

$$
\begin{array}{rlrl}
\mathcal{O}_{1} & =\Phi_{1} \Phi_{2}, & & \mathcal{O}_{2}=\Phi_{3} \Phi_{4}, \\
\mathcal{O}_{3}=\Phi_{1} \Phi_{4}, & & \mathcal{O}_{4}=\Phi_{2} \Phi_{3} .
\end{array}
$$

The lowest dimension terms which are $R_{p}$ invariant and phase dependent arise as soft SUSY breaking $A$-terms and 
appear in the scalar potential as

$V=\sum_{i=1}^{4} \frac{m_{i}^{2}}{2}\left|\Phi_{i}\right|^{2}+\frac{A_{1}}{8} \frac{m_{s}}{M} \mathcal{O}_{1}^{2}+\frac{A_{2}}{8} \frac{m_{s}}{M} \mathcal{O}_{2}^{2}+\frac{A_{3}}{8} \frac{m_{s}}{M} \mathcal{O}_{3}^{2}$

$+\frac{A_{4}}{8} \frac{m_{s}}{M} \mathcal{O}_{4}^{2}+\ldots$, where the ellipses stand for other terms of the same order with different products of the gauge invariant operators, loop induced contributions, and higher order terms in the $(1 / M)$ expansion. Substituting the vevs given in Eq. (34) we generate the potential terms given in Eq. (37).
[1] T. Gherghetta, C. F. Kolda, and S. P. Martin, Nucl. Phys. B468, 37 (1996).

[2] For a review, see K. Enqvist and A. Mazumdar, Phys. Rep. 380, 99 (2003).

[3] I. Affleck and M. Dine, Nucl. Phys. B249, 361 (1985).

[4] A. D. Linde, Phys. Lett. B 160, 243 (1985).

[5] M. Dine, L. Randall, and S. D. Thomas, Phys. Rev. Lett. 75, 398 (1995).

[6] M. Postma and A. Mazumdar, J. Cosmol. Astropart. Phys. 01 (2004) 005.

[7] N. Shuhmaher and R. Brandenberger, Phys. Rev. D 73, 043519 (2006).

[8] See e.g.., R. Allahverdi, K. Enqvist, J. Garcia-Bellido, and A. Mazumdar, Phys. Rev. Lett. 97, 191304 (2006).

[9] R. Allahverdi and A. Mazumdar, arXiv:hep-ph/0603244.
[10] K. A. Olive and M. Peloso, Phys. Rev. D 74, 103514 (2006).

[11] R. Allahverdi and A. Mazumdar, arXiv:hep-ph/0608296.

[12] T. W. B. Kibble, Phys. Rev. 155, 1554 (1967).

[13] H. P. Nilles, M. Peloso, and L. Sorbo, J. High Energy Phys. 04 (2001) 004.

[14] R. Casadio, P. L. Iafelice, and G. P. Vacca, arXiv:hep-th/ 0702175.

[15] J. H. Traschen and R. H. Brandenberger, Phys. Rev. D 42, 2491 (1990).

[16] L. Kofman, A. D. Linde, and A. A. Starobinsky, Phys. Rev. D 56, 3258 (1997).

[17] Y.B. Zeldovich and A. A. Starobinsky, Zh. Eksp. Teor. Fiz. 61, 2161 (1971) [Sov. Phys. JETP 34, 1159 (1972)]. 